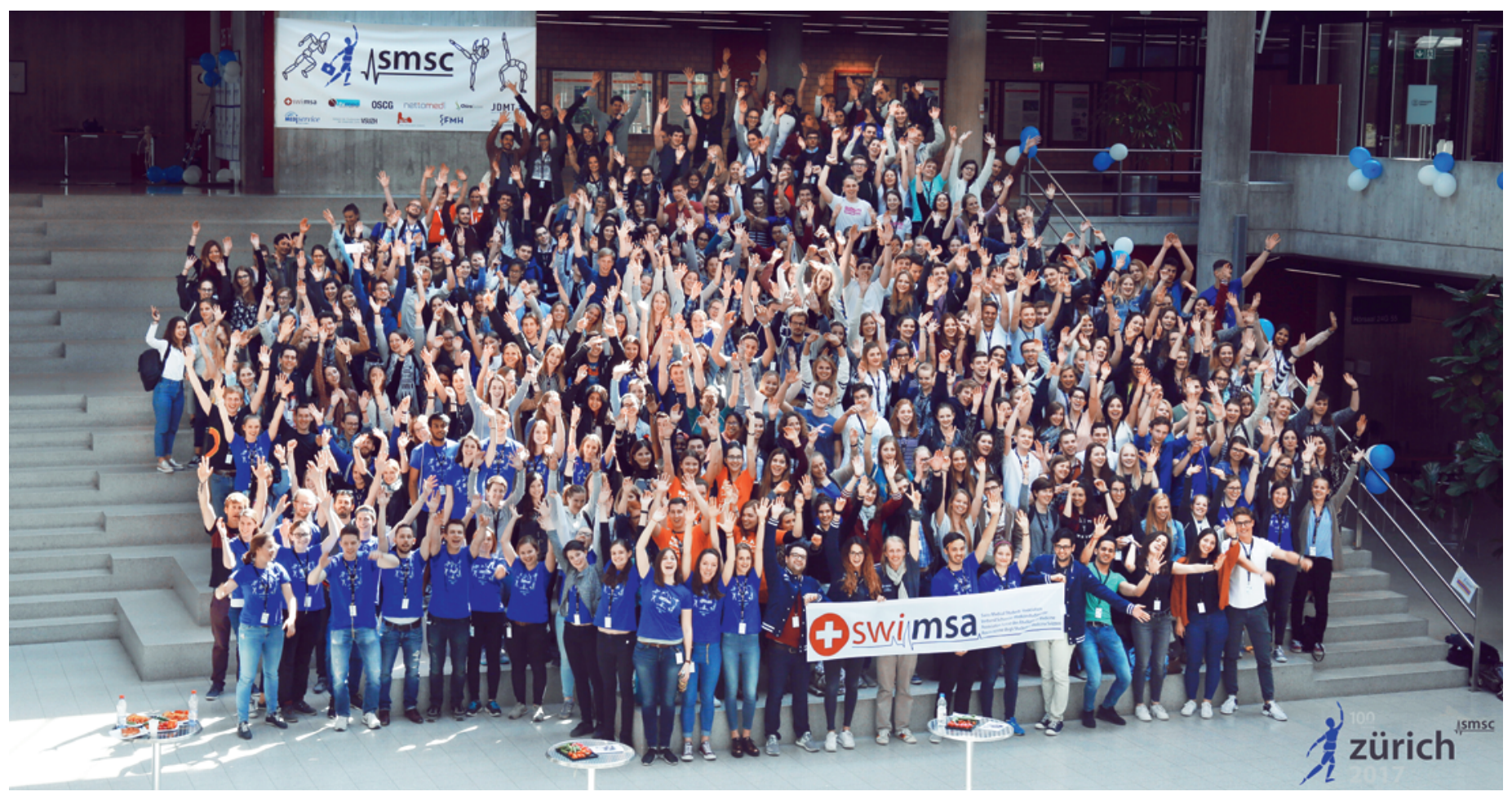

\title{
La relève suisse en mouvement
}

\section{Bea Albermann}

swimsa Liaison Officer FMH

C'est sous le thème «Let's get moving!» que se sont rassemblés en avril quelque 400 étudiants en médecine de toute la Suisse à l'occasion de la Swiss Medical Students' Convention (SMSC) qui s'est tenue à Zurich. Cela fait déjà 100 ans que les étudiants suisses s'engagent activement dans des projets sociaux en dehors du cadre académique, apportant ainsi une contribution importante à la formation des médecins.

Qu'il s'agisse d'intervenir dans les milieux scolaires, de soutenir des projets de développement à l'autre bout du monde ou de rassembler des donneurs de cellules souches sanguines, des étudiants en médecine de toute la Suisse s'engagent bénévolement en dehors de leurs études pour attirer l'attention sur la santé de la société dans le cadre de la Swiss Medical Students' Association (swimsa).

C'est par exemple le cas d'un groupe de 20 étudiants de Zurich qui a organisé en avril la Swiss Medical Students' Convention (SMSC) - un congrès pour étudiants en médecine qui a lieu deux fois par an dans une autre université et sur un thème spécifique.
Quelque 350 étudiants de toute la Suisse ont participé à ce week-end consacré cette année à la médecine du sport et à la réadaptation. Des discussions interactives, des exposés passionnants et des ateliers pratiques leur ont permis d'accroître leurs connaissances.

\section{Priorité à l'apprentissage pratique}

Contrairement aux études de médecine remplies de cours magistraux théoriques et abstraits, les weekends de la SMSC offrent aux étudiants la possibilité de mettre directement en pratique les connaissances acquises: du diagnostic par ultrason après un accident 
aux principes de base de la physiothérapie et de la chiropractie en passant par les troubles liés au sport ou à l'alimentation, la SMSC de Zurich a couvert de nombreux domaines de la médecine du sport et de la réadaptation qui ne sont pas abordés dans les cours habituels. Des questions de santé publique, par exemple le rôle de l'activité physique dans la médecine de premier recours, ou des techniques de motivation à l'activité physique à l'intention des médecins de famille ont également été thématisées.

\section{Inspiration grâce aux spécialistes et experts}

Les étudiants ne sont pas les seuls à s'engager à la SMSC: les ateliers et les exposés sont animés par des spécialistes bénévoles. Plus ces derniers seront motivés à parler de leur spécialisation, plus ils pourront enthousiasmer et inspirer leur auditoire. Les domaines médicaux moins populaires ou familiers ont ainsi l'opportunité d'interpeller immédiatement la relève et de lui ouvrir de nouvelles options. Du développement de prothèses high tech à l'EPFZ à la médecine du sport pédiatrique, le congrès a présenté un large éventail de possibilités de carrière dans le cadre d'un échange direct et immédiat avec les étudiants. Le réseautage entre ces futurs confrères était également au rendezvous: pour les étudiants, la SMSC offre l'occasion idéale d'échanger, de découvrir des projets et de nouer de nouveaux contacts.

\section{Echange et réseautage sont essentiels pour travailler dans le secteur de la santé}

L'importance fondamentale des échanges et des réseaux est démontrée chaque année par la réunion de

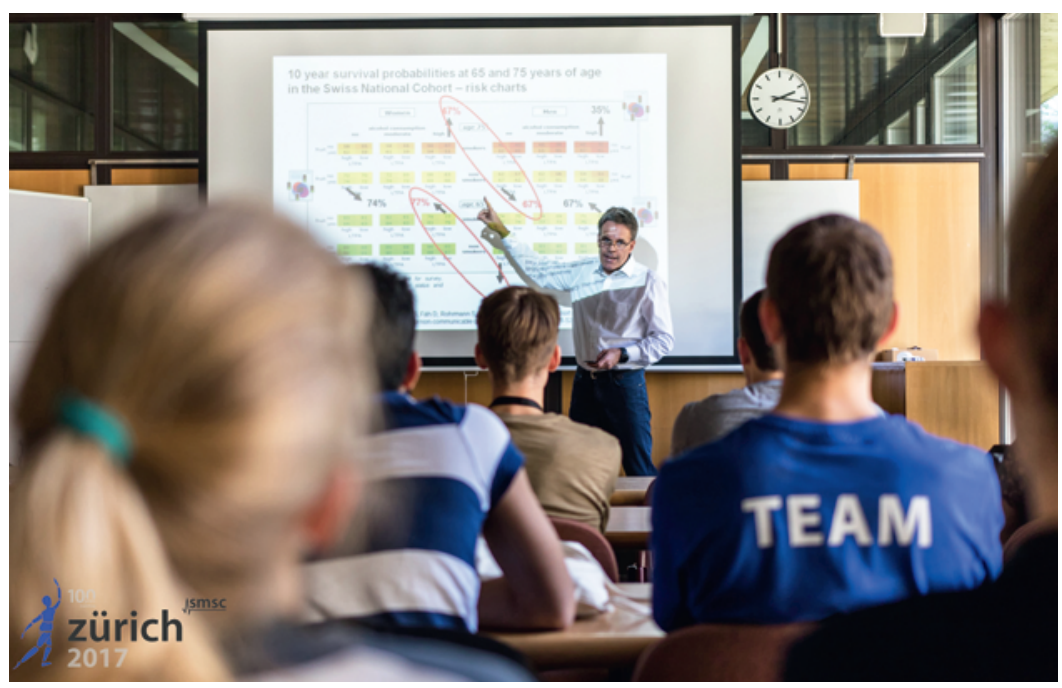

Les ateliers et les exposés sont animés par des spécialistes bénévoles.

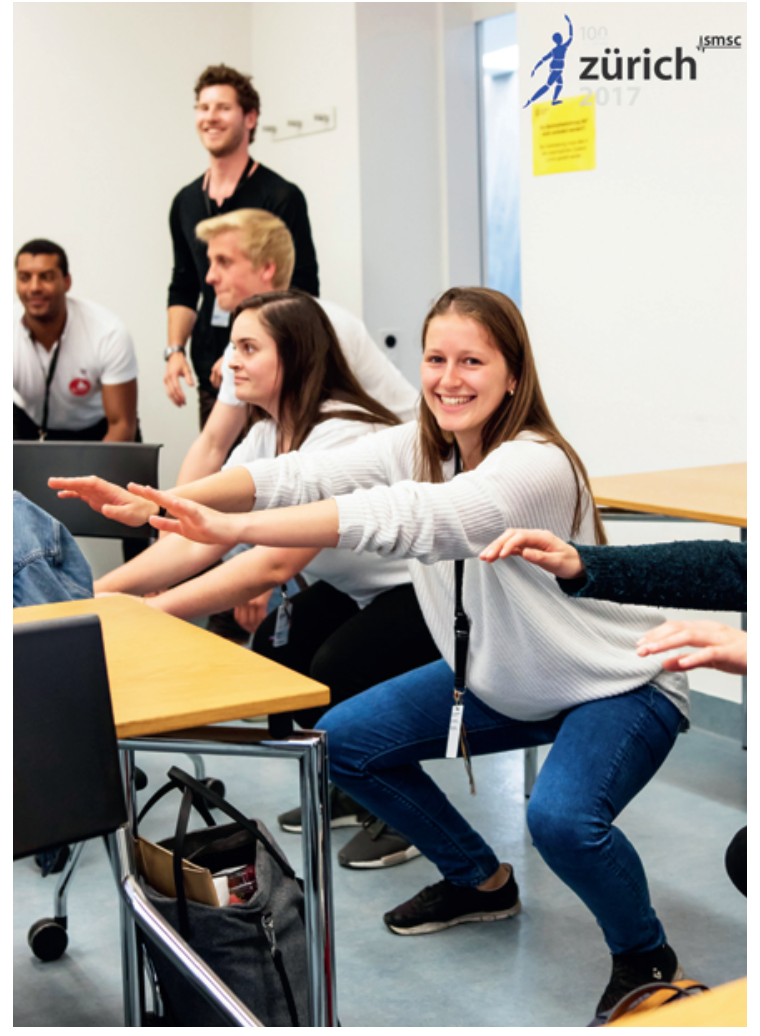

Mettre directement en pratique les connaissances acquises: le week-end SMSC de Zurich a couvert de nombreux domaines de la médecine du sport et de la réadaptation qui ne sont pas abordés dans les cours habituels.

l'International Federation of Medical Students' Association (IFMSA) - une sorte de SMSC au niveau international. A cette occasion, des étudiants en médecine du monde entier, dont une délégation suisse, participent à des idées et projets innovants visant une société durable et en bonne santé ou une meilleure formation des médecins. Les problèmes locaux étant très différents selon le continent - du travail d'information de base au Ghana aux cours de radiologie au Canada -, les différentes régions du monde peuvent ainsi bénéficier mutuellement de réflexions et de stratégies de solution. Sans un échange actif, ce progrès ne serait pas concevable. C'est par exemple le cas du «Project Fair» de la SMSC qui permet à diverses organisations de se présenter par le biais d'un stand et d'entrer ainsi directement en contact avec les étudiants.

\section{Potentiel de soutien pour un engagement extraordinaire}

L'organisation d'un congrès d'une telle ampleur parallèlement à des études de médecine est loin d'être évidente. Le comité d'organisation à Zurich qui a pu compter sur l'aide d'une cinquantaine d'étudiants a également été tributaire du soutien d'entreprises et 


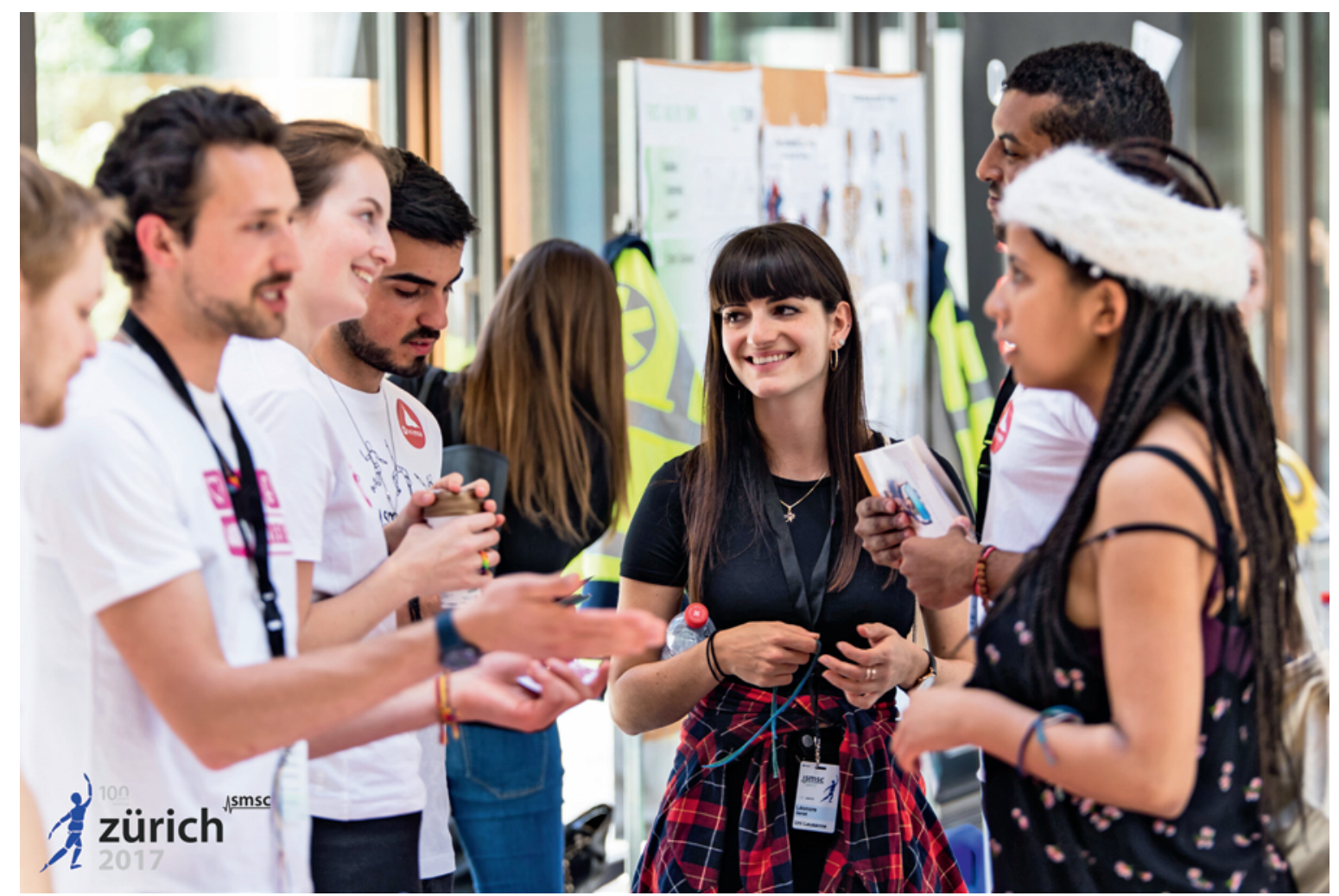

Les différents stands permettent aux étudiants de nouer des liens et d'échanger avec des organisations médicales, des futurs employeurs et de découvrir des projets bénévoles.

d'organisations professionnelles. Pour continuer à l'avenir à organiser de tels événements, la swimsa est continuellement en quête de financement auprès d'organisations externes. Et cela porte ses fruits: depuis un siècle exactement, la swimsa est une inspiration pour les futurs médecins qu'elle soutient dans leur cursus professionnel. Au vu du nombre toujours croissant des étudiants en médecine, l'engagement de la swimsa contribue ainsi de manière déterminante à la qualité des médecins de demain. Ce n'est qu'ensemble que l'on peut faire bouger les choses - et comme chacun sait, bouger est bon pour la santé!

\section{Crédit photos}

(1) swimsa

\section{Complément d'information:}

La prochaine SMSC aura lieu à Bâle du 27 au 29 octobre 2017 et sera consacrée au thème de la médecine forensique. Vous trouverez de plus amples informations sur notre site: www.swimsa.ch/fr. Si vous êtes intéressé par une collaboration avec la swimsa ou si vous avez des questions concernant les projets des étudiants, nous sommes à votre disposition à I'adresse suivante: contact[at]swimsa.ch. 